\title{
A Conventional Model for Security Challenges in Industrial Internet of Things
}

\author{
Mr.D.Vinod ${ }^{1}$ M.E, Mrs.V.Subapriya ${ }^{2}$ M.E \\ Assistant Professor, Dept of Information Technology, Mohamed Sathak A J College of Engineering, Chennai-603103 ${ }^{1}$ \\ Assistant Professor, Dept of Information Technology, Mohamed Sathak A J College of Engineering, Chennai-603103 ${ }^{2}$
}

\begin{abstract}
Web of things has been broadly associated for home, industry, and various distinctive applications. For these applications, secure information transmission transforms into an essential issue to ensure the structure prosperity. Mixed encryption technique is another cryptographic perspective and it can be associated with the Internet of Things. It gives the benefit of the symmetric key and unbalanced key execution. It engages strong security and low computational flightiness. The proposed procedure mulls over that a cream encryption figuring which has been coordinated remembering the true objective to diminish risks and redesigning encryption's speed and less computational unpredictability. The inspiration driving this cross breed computation is information uprightness, arrangement, nonrevocation in data exchange for Internet of things.
\end{abstract}

Keywords: Web of Things (IOT), Digital Signature Algorithm (DSA), AES Algorithm

\section{INTRODUCTION}

Web of Things (IOT) is another framework that's being employed by remote sensing element affiliations and radio repeat ID (RFID) through remote framework and development to attain general impression of data, sturdy transmission and wise taking care of. so guaranteeing security and prosperity square measure the key options of IOT. This security is said to call data (RFID), remote correspondences data security, organize transmission of data security, insurance and security data coming up with security. Likewise it's basic to possess targeted examination and analysis on set up and alter of security problems in IOT. The quick amendment of security and insurance in vast scale square measure the determinant factors of IOT. The essential inspiration driving framework security and knowledge protection is to attain mystery and dependableness. within the past few decades, the PC, the net and convenient web advancement has passed on uncommon changes to human culture, with the amendment of data trade and correspondence between people. the quality machines while not the net affiliation cannot meet our application needs. omnipresent accessibility propels the amendment of data correspondence among things and things. within the past few years, the net of things has been extensively related to trade, tries, affiliations, school, national security, and our step by step life. From the angle of building, the net of things will be usually secluded into 3 layers, to be explicit acknowledgment layer, planned out layer, and application layer. The acumen layer changes the data of things to the clear modernised flags through RFID, sensors, et cetera. On the opposite hand, the framework layer transmits these propelled signs to gazing stages by strategies for a connected organize. Finally, the appliance layer unscrambles and applies propelled signals through relating programming. Among these 3 layers, security is a difficulty to make sure signal is cured accumulated, transmitted, and deciphered by the applications. each the quality center points and sink centers square measure defenseless against a grouping of security attacks, to Illustrate, refusal of organization strikes, or unlawful management and frustration. These ambushes might deal the sensitive data and lead to breakdowns. on these lines data security should be approved for data genuineness, certainly, and assurance. cryptography may be a needed progress for the safety of the net of things. The Advanced cryptography commonplace (AES) and error correction code (Elliptic Curve Cryptography) square measure wide used for data security. It uses the elliptic twists as leading edge check, and therefore the speed of mechanized stamp and affirmation to speedier than DSA(Digital Signature Algorithm), and AES computation cryptography information is obvious, quick and robust. The cream key development, that is bothered the characteristics of regular key and uneven key, is effecting increasingly revered. In any case, the employment of the net of things continues to be within the examination organize. In any case, there square measure still problems on the cryptography organize for once the cryptography computation is related to retiring contraptions of the net of things. visible of low machine resources, the way to outfit solid security with low machine versatile quality is making an attempt. during this paper, we tend to address this issue and propose a mixed cryptography count to effectively use the heterogeneous limits of the frameworks organization elements within the web of things systems. The sensing element center points will use the gear cryptography chip whereas people by and huge key institution PKI and mystery word development and alternative explicit suggests that ensures the safety and prosperity for center points to accumulate 
data. Our approach empowers cream cryptography to attain a predominant sensible on the data security whereas the data is transmitted over variety

\title{
II. ENCRYPTION ALGORITHMS
}

\begin{abstract}
AES Algorithm: AES run fuses a high security, helpful is unadulterated programming pack use, the speed is likewise immediately, and AES is incomprehensibly low memory necessities, all together that it's a lot of fitting for several limited setting. AES encoding procedure is in an exceedingly four $*$ ( $\mathrm{Nb}$ is up to the information piece length parceled by thirty two, the quality AES is four bytes) undertaking of matrix, the system is besides suggested as the "express", its fundamental worth may be a plaintext thwart (a PC memory unit the span of the grid is unmistakably a segment inside the square). Encryption comprises of the following steps:
\end{abstract}

1) Add Round Key: Each PC memory unit inside the network and thusly the key age subject inside the current round of the key age subject to come up with the XOR assignment.

\section{DESIGN AND APPLICATION OF HYBRID KEY TECHNOLOGY IN THE SYSTEM}

A. Mixed Cryptography sort out information security transmission style inside the issue Three-layer arrangement is normal in wander with the web of things, information by identifying contraptions to get the material activity layer, so sent to the obtainment terminal, so moved into the prosperity structure. start of all, through the separation module process once puzzle making process, so through the clever correspondence interface module trades module information transmission to the framework layer, \{the information $\mid$ the info $\mid$ the information $\}$ is transmitted to the sharp correspondence interface module of the applying layer data, finally once secret creating, disconnection was unavoidably server gets. Frameworks organization data security transmission consolidates information blend handset structure, shrewd correspondence interface plot, information security scheme. the info | the knowledge | the data security contrive is used to insist the transmission prosperity perception information; in the essential used for information transmission choice and learning causation and tolerating. the most contains inside and outside framework process unit, organize segregation module, data puzzle creating and riddle making module, affirmation module. Encryption, information security contrive secret creating, affirmation server sort out process unit external process unit of savvy correspondence interface unit $\backslash$ two-way unidirectional separation channel.

\section{Chapter-I}

1.1.Literature Survey: The Internet of Things (IoT) could be a dynamic overall data sort out involving Internet connected objects, like repeat identi_cations, sensors, and actuators, yet as different instruments ANd extraordinary machines that are getting an irreplaceable bit of the web. Over the recent years, we have seen an extremity of IoT game plans making their approach into the trade business focus. Setting careful correspondences and handling have endeavor a basic part all through the recent years of present enrolling and district unit expected that would accept a signi_cant part inside the IoT perspective yet. in the midst of this paper, we tend to review a spread of standard and imaginative IoT game plans the extent that setting careful advancement sees. additional on a very basic level, we tend to judge these IoT courses of action using a framework that we tend to laid out around without a doubt comprehended contextaware handling theories. This audit is expected to work a proposal and a theoretical structure for setting careful headway and examination inside the IoT perspective. It besides gives a coherent examination of existing IoT thing inside the business focus and highlights arrangement of probably signi_cant examination orientation and examples.

Title: A Survey on Internet of Things From

Industrial Market Perspective.

Author: C. H. Liu (chiliu@ bit.edu.cn)

Year:2014

\section{Chapter-2}

1.2 Literature survey: With the affirmation of sensor-rich PDAs (e.g., sensible phones and wearable contraptions), Mobile Crowdsourcing (MCS) has created as a beneficial logic for learning assortment and process. Differentiated and old-fashioned Wireless locator Networking (WSN), MCS holds a couple of blessings like quality, quantifiability, costcapability, and human understanding. Regardless, MCS still faces a couple of challenges with respect to security, assurance and trust. This paper gives an outline of those troubles and discusses potential courses of action. we tend to look at the characteristics of MCS, choose its security perils, and depiction fundamental necessities on an ensured, security defending and reliable MCS system. Further, we tend to study existing game plans maintained these necessities and dissect their administrators and cons. Finally, we have a tendency to demonstrate open issues and propose some future examination heading. 
Title: A Survey on Security, Privacy and Trust in Mobile Crowdsourcing Author :Wei Feng, Zheng Yan,Akulwar.

Year:2017

\section{Chapter-3}

\subsection{Literature survey:}

Advances in data and correspondence developments have LED to the ascent of web of Things (IoT). inside the care condition, the utilization of IoT propels passes on solace to specialists and patients as they will be associated with contrasted remedial areas, (for instance, consistent time allotment observation, diligent data organization, restorative emergency organization, blood information organization, and prosperity organization). The radio-repeat conspicuous evidence (RFID) development is one among the middle progressions of IoT associations inside the care condition. To satisfy the diverse security needs of RFID advancement in IoT, a couple of RFID affirmation designs are foreseen inside the earlier decade. Starting late, elliptic curve cryptography (ECC)- based RFID confirmation designs have pulled in stores of thought and are utilized as a part of the care condition. in the midst of this paper, we tend to discuss the affirmation needs of RFID approval designs, and particularly, we tend to gift an overview of ECC-based RFID affirmation contrives similar to execution and security. regardless of the way that a vast part of them can't satisfy all security needs and have elegant execution, we have a tendency to build up that there ar 3 starting late foreseen ECCbased affirmation designs appropriate for the care surroundings to the extent their execution and security.

Title: An Analysis of RFID Authentication Schemes for Internet of Things in Healthcare Environment Using Elliptic Curve Cryptography

Author: Debiao He and Sherali Zeadally

Year:2015

\section{Chapter-4}

1.4 Literature survey: Certain relational collaboration (PSN) reinforces minute social activities wherever and at whatever point with the assistance of heterogeneous frameworks. keeping in mind the end goal to spare assurance and win tried and true PSN, baffling affirmation on center trust is predicted in PSN. In any case, the written work still needs honest to goodness examinations on this issue. in the midst of this paper, we have a tendency to propose relate degree strange approval theme for affirming each pseudonym trust levels to help tried and true PSN with assurance protecting. The subject achieves secure puzzling affirmation with obscurity and unforeseen traceability on the likelihood of a trusty expert (TA). By applying a go down answer, it will guarantee correspondences among center points for relate degree widened time span even once the metal isn't out there. likewise, crafted by cluster signature affirmation additional declines the cost of genuineness check of an outsized extent of messages. Execution examination and examination additional exhibit that the foreseen point is effective with significance security protection, computation multifaceted nature, correspondence regard, flexibility, reliability, and quantifiability.

Title: Anonymous Authentication for Trustworthy Pervasive Social Networking.

Author: Zheng Yan, Senior Member,

Year:2016

\section{EXISTING PROBLEM}

The essential huge injury of mixed encoding condition supported out of date cryptography is unlawful sharing of key among sender and recipient, i.e. key course drawback Second the memory assess that insinuates the measure of accomplice degree encoded message and thusly the key size ought to try and be lessened. i.e. key organization drawback. The issue related to oftenness ID is revealing the sensitive information could achieve security break issues. the inconvenience related to the mixed encoding framework is that it doesn't offer security, once the information is unscrambled.

\section{PROPOSED SYSTEM}

Cross breed coding condition improves the speed of coding and coding and it additionally fathoms the key organization drawback. Cross breed crypto graphical framework contains in having only the people from a given gathering arranged to sign the messages changed among the bundle. The objective part will be set up to check if a stamp is significant, while not revealing truth identity of the financier. 


\section{PERFORMANCE ANALYSIS}

Hybrid Cipher Algorithm in non symmetric cryptography and symmetric cryptography in one, with high security and fast speed, small storage space, more suitable for Internet of things some limited environment in such.
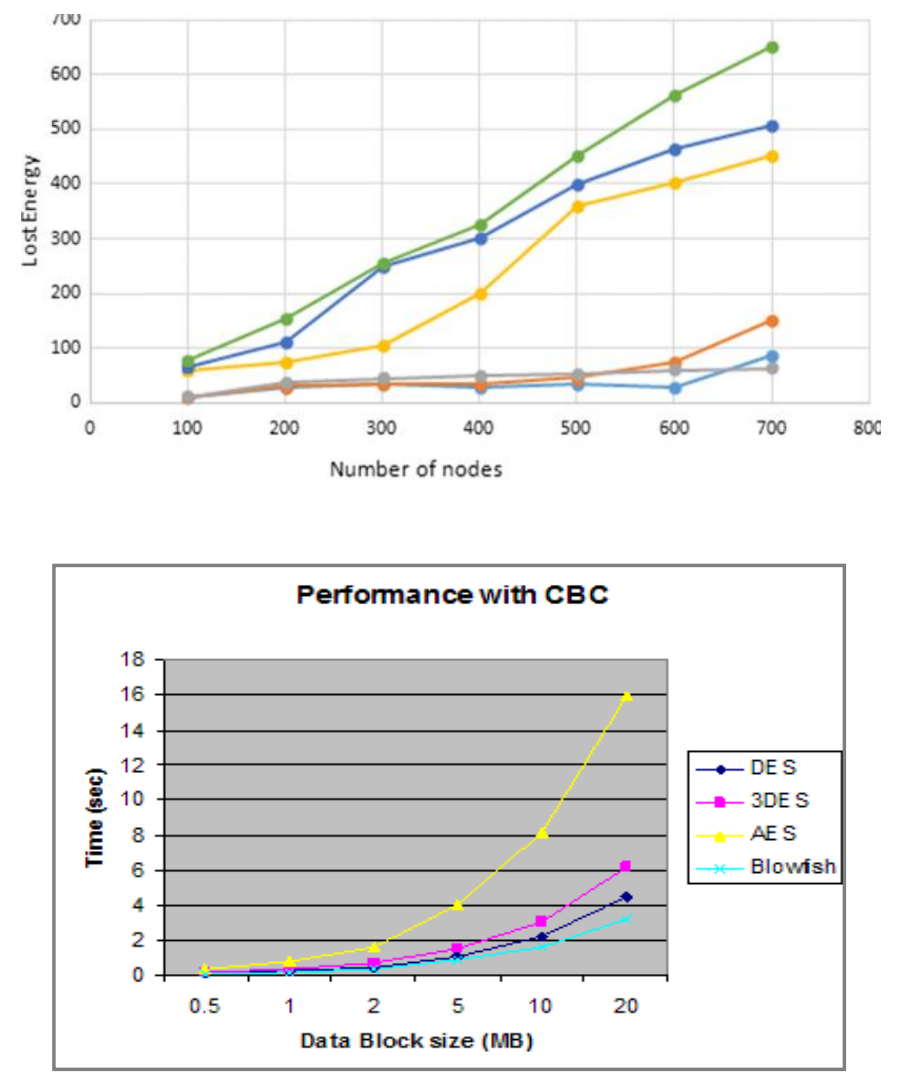

\section{CONCLUSION}

In this unique duplicate, we've offered the perceived issues identified with guaranteeing message trustworthiness and approval by prescribes that of automated imprints among the setting of disseminate/ purchase in organizations. The before long conceivable courses of action require essentialness power and quantifiability, that square measure principal necessities inside the setting of business IoT; besides, they mishandle the secrecy and decoupling properties for event see in disperse purchase in plans. To deal with these issues, we've foreseen a pack signature-based point besides, associated it to a perspective of establishment less point based convey/purchase in advantage for sensors. we've through test observation assessed it therefore on encounter the resultant execution heightening and thusly the development inside the battery usage. An open issue in our approach is that the key repudiation, generally related to a distributer abuse the cluster. In our approach, we have gotten the basic assurance from [63], where the stamping and check parameters, severally gpk and gsk[i] for the I-th distributer and gpk for the supporters are balanced and retransmitted once a center point gets out. Regardless of having a straightforward use, such an answer isn't perfect since the related regard (the extent that foreswearing time and essentialness usage) is wide. As a future work we tend to will inquire about a huge amount of legitimate renouncement designs among the ones inside the present written work, and alter it in our approach; in development, unmistakable check designs fitting for our focuses, for instance, bunch marks [64] or ring-based ones [65], are mulled over.

\section{REFERENCES}

[1]. L. Atzori, A. Iera, and G. Morabito, “The internet of things: A survey,” Computer Networks, vol. 54, no. 15, pp. 2787-2805, 2010.

[2]. A. V. Dastjerdi and R. Buyya, "Fog Computing: Helping the Internet of Things Realize Its Potential," Computer, vol. 49, no. 8, pp. 112-116, August 2016.

[3]. W. Feng, Z. Yan, H. Zhang, K. Zeng, Y. Xiao, and T. Hou, “A Survey on Security, Privacy and Trust in Mobile Crowdsourcing," IEEE Internet of Things Journal, vol. In Press, 2017. 


\section{IJARCCE}

Vol. 7, Issue 11, November 2018

[4]. P. Eugster, P. Felber, R. Guerraoui, and A.-M. Kermarrec, "The many Faces of Publish/subscribe,” ACM Computing Surveys, vol. 35, no. 2, pp. 114-131, June 2003

[5]. A. Al-Fuqaha and M. Guizani and M. Mohammadi and M. Aledhari and M. Ayyash, "Internet of Things: A Survey on Enabling Technologies, Protocols, and Applications," IEEE Communication Surveys \& Tutorials, vol. 17, no. 4, pp. 2347-2376, Fourth Quarter 2015.

[6]. C. Esposito and A. Castiglione and F. Palmieri and M. Ficco and K. K. R. Choo, "A Publish/Subscribe Protocol for Event-Driven Communications in the Internet of Things," in Proceedings of the IEEE 14th International Conference on Dependable, Autonomic and Secure Computing, August 2016, pp. 376-383.

[7]. Y. Koren, The Global Manufacturing Revolution: Product-Process- Business Integration and Reconfigurable Systems. Wiley, June 2010.

[8]. M. Wollschlaeger and T. Sauter and J. Jasperneite, "The Future of Industrial Communication: Automation Networks in the Era of the Internet of Things and Industry 4.0," IEEE Industrial Electronics Magazine, vol. 11, no. 1, pp. 17-27, March 2017.

[9]. EFFRA, "Factories Of The Future - Multi-annual roadmap for the contractual PPP under Horizon 2020," https://ec.europa.eu/research/industrial technologies/factories-of-thefuture en.html, accessed: 2016-10-24.

[10]. Executive Office of the President President's Council of Advisors on Science and Technology, "Report To The President On Capturing Domestic Competitive Advantage In Advanced Manufacturing," http://energy.gov/eere/downloads/report-president-capturingdomesticcompetitive- advantage-advanced-manufacturing, accessed: 2016-10-24.

[11]. T. Sauter, "Public discussion: Future trends in factory communication systems," in Pro Sixth IEEE Int. Workshop Factory Communication Systems (WFCS), May 2006.

[12]. T. Sauter, "The three generations of field-level networks-Evolution and compatibility issues," IEEE Trans. Ind. Electron., vol. 57, no. 11, pp. 3585-3595, Nov. 2010

\section{BIOGRAPHIES}

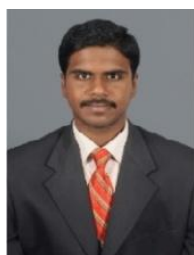

D. Vinod received the bachelor's degree in 2010 in computer science from Anna University in Asan Memorial College of Engineering and Technology. He finished his master's degree in 2012 in computer science from Anna University in S.A. Engineering College. His research interests include design and analysis of algorithms concerning wireless networks, network security, Internet of things, etc. Topics include coverage problems in sensor network, routing, top-k query, capacity (throughput) study, diagnosis of WSN, and so on. He is a member of the IEEE.

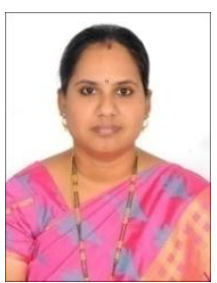

V. Subapriya received the bachelor's degree in 2006 in Computer Science from Anna University Affiliated College. Master's in Computer Science in 2015 from Sathyabama University and secured Gold Medal for the same. Her area of interest includes Cloud Computing, IoT and Network Security. She is the member of IEEE and IAENG. 